\title{
Cryosurgery for trichiasis in black patients
}

\author{
DAVID A PEART AND JOHN C HILL
}

From the Department of Ophthalmology, Groote Schuur Hospital and University of Cape Town, South Africa

SUMMARY A total of 34 lids with aberrant lashes were treated with the specifically designed Collin cryoprobe without microthermocouple temperature monitoring. A high success rate of $82 \%$ was obtained. By applying the cryoprobe to the lid margin and conjunctival surface instead of to the skin it was possible to limit the degree of depigmentation in these highly pigmented lids, and only one patient showed a mild cosmetic blemish. The treatment was repeated on up to three occasions without an increase in complications.

Cryotherapy has become accepted as a successful form of treatment in trichiasis, ${ }^{1-4}$ especially if a large number of aberrant lashes are present. ${ }^{5}$ The initial experimental work and clinical trials were described by Sullivan, Beard, and Bullock in 1976. ${ }^{1}$ Since then numerous trials have demonstrated the efficacy of this mode of treatment and its advantages over epilation, electrolysis, radiotherapy, and surgery..$^{1-4}$

Cryotherapy has not been used extensively in black patients because of the marked susceptibility of pigment cells to freezing and the subsequent problem of depigmentation. ${ }^{5}$ Not only does this cause a cosmetic blemish but in patients living in hot climates there is the risk of actinic damage and its sequelae. This is unfortunate, as many of the people living in underprivileged parts of the world, with exposure to conditions such as trachoma, are dark skinned. The use of cryotherapy, which is easy to administer and not expensive compared with surgery, would offer many advantages. This paper describes a method of using cryotherapy to treat trichiasis in heavily pigmented lids, without using thermocouple control and with the avoidance of significant depigmentation. Many of the patients were treated on more than one occasion without significant adverse effects.

\section{Material and methods}

All procedures were performed in the minor outpatient theatre. Local anaesthesia was obtained with benoxinate $2 \%$ drops into the conjunctival sac and local infiltration of the lid with $2 \%$ lignocaine with adrenaline $(1: 100000)$. A plastic spoon was

Correspondence to Dr J C Hill, Department of Ophthalmology, Medical School, University of Cape Town, Observatory 7925, Cape Town, South Africa. positioned to protect the globe. The Collin cryoprobe attached to the Spembly-Amoils BMS-40 unit was used in this series with nitrous oxide as the cooling agent. Previous workers used a double freeze-thaw cycle whereby the probe was applied once to the conjunctival surface and once to the skin surface. This direct contact with skin incorporates a large area of skin within the iceball (Fig. 1A). We modified this technique by using a double freeze-thaw cycle applying the probe to the conjunctival surface and then to the lid margin (Fig. 1B). This prevents direct freezing of the skin and limits the area of skin incorporated into the iceball. The duration of application was 20 seconds to the lower lid and 25 seconds to the upper lid. It has been shown ${ }^{2}$ that with this probe the desired temperature of $-20^{\circ} \mathrm{C}$ can be consistently obtained at the level of the hair follicle if these time periods are used. A thermocouple was therefore not used to monitor the temperature. The lashes were not epilated. Chloromycetin ointment was applied and the eye padded for 24 hours. Patients were followed up at two weeks and again at six weeks after treatment. Thereafter they were seen when necessary or at three-monthly intervals. Any remaining lashes were epilated at the first post-treatment check, and, if aberrant lashes were found to have persisted or recurred at the second or subsequent visit, the patients were treated again in an identical fashion to that described above.

\section{Results}

Twenty-three patients and a total of 34 lids were treated. The causes of the trichiasis are given in Table 1. No pathological cause could be found in 27 lids. Many of these patients suffered from severe diffuse 

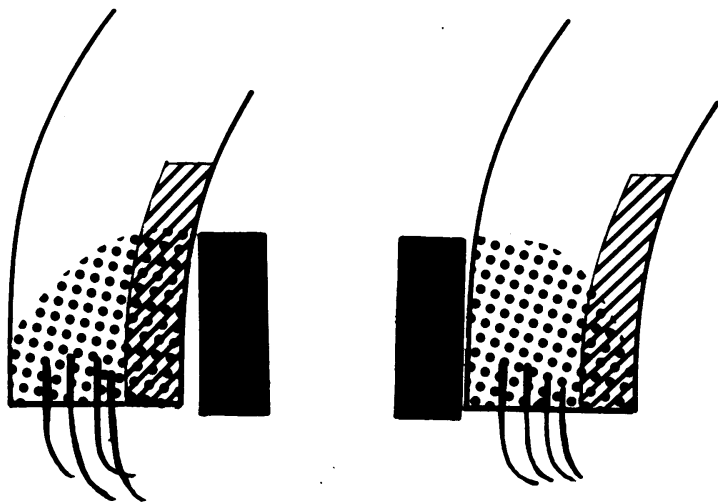

Fig. 1A Usual method of applying the cryoprobe. A double freeze-thaw cycle is performed, the probe being applied in turn to the conjunctival and to the skin surfaces of the lid.

During the latter application the iceball (stippled) involves a large area of skin.

aberrant lashes, especially laterally, and this would appear to be a local genetic characteristic. The lower lid was affected in only four cases.

The median length of follow-up was 13 months, with a range of two to 26 months.

The results of treatment are shown in Table 2. Six patients (six lids) were excluded from the study because they were lost to follow-up. The criterion for successful treatment was elimination of aberrant lashes in the treated area with relief of symptoms. With repeated treatments the success rate was $82 \%$ ( 23 of 28 lids). Four patients showed some improvement after one application but were not treated further for various reasons. In one instance the machine was not working and the patient was subsequently lost to follow-up, two patients were referred for surgery because of concomitant dermatochalasis with entropion, and only one patient refused further
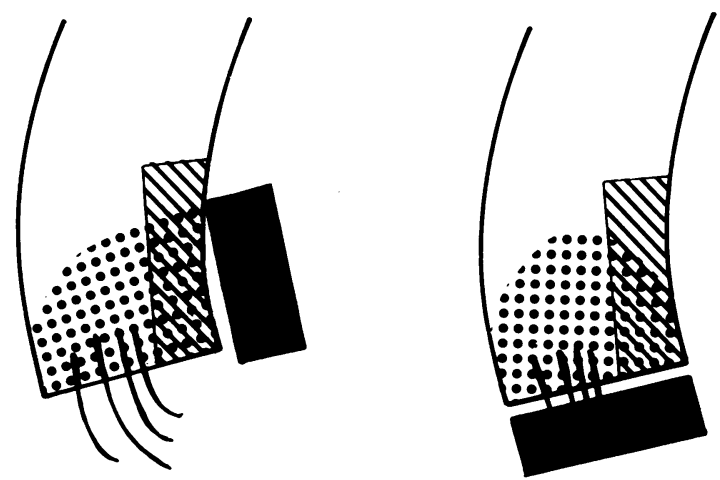

Fig. 1B In our patients the cryoprobe was applied to the conjunctival surface and to the lid margin. The iceball (stippled) incorporates the lash follicles, but skin involved is kept to a minimum. treatment. One patient showed no improvement after a single treatment.

The incidence of significant complications was low. Lid swelling occurred in all patients immediately after treatment but was usually of only moderate severity with mild discomfort. It settled within a week. Two patients developed lid notching after treatment; in both cases this followed a single application.

Lid depigmentation was graded into three groups: (O) no depigmentation or visible only with magnification; (I) Involving only the lid margin and not cosmetically objectionable (Fig. 2); (II) involving lid skin. Nineteen lids showed grade I depigmentation and one patient grade II depigmentation. The remaining eight lids had grade $\mathrm{O}$ depigmentation only.

Six patients subsequently developed trichiasis in untreated areas. In four cases this was adjacent to the treated area and in two cases in another lid.

\section{Discussion}

Pigment cells are extremely susceptible to freezing and can be destroyed at temperatures of $-10^{\circ} \mathrm{C} .^{6}$ Hair follicles are destroyed at temperatures of $-20^{\circ} \mathrm{C}$. It has been stated that depigmentation will always occur if trichiasis is effectively treated and that therefore this mode of treatment is not advised in black patients. ${ }^{5}$ We believe that in reducing the degree of freezing of the lid skin by applying the probe as described above it is possible to reduce the amount of depigmentation to an acceptable level and still obtain satisfactory results.

This study demonstrates the successful use of cryotherapy to treat trichiasis without temperature monitoring by using a standardised probe for set time periods. This is important because thermocouples are expensive and being delicate instruments require

Tabe 1 Causes of trichiasis

\begin{tabular}{lr}
\hline Idiopathic & 27 \\
Trachoma & 3 \\
Trauma & 3 \\
Blepharitis & 1 \\
\hline
\end{tabular}

Table 2 Results of treatment

\begin{tabular}{|c|c|c|}
\hline & \multicolumn{2}{|c|}{ No. of lids } \\
\hline Lost to follow-up or deceased & 6 & \\
\hline Success with 1st application & $11)$ & \\
\hline 2nd application & 9 & 23 \\
\hline 3rd application & 3 & \\
\hline Improvement without eradication of aberrant lashes & 4 & \\
\hline No improvement & 1 & \\
\hline
\end{tabular}


Fig. 2 Grade I depigmentation. Only the lid margin skin is involved.

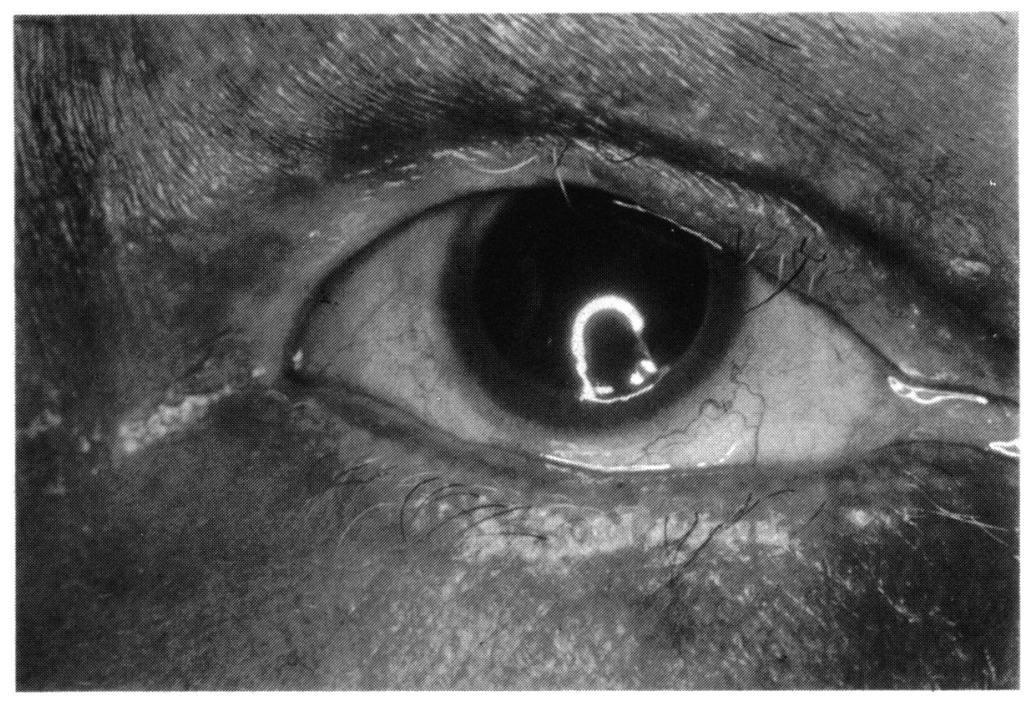

much care in their use. The absolute necessity of using them would restrict the scope of cryotherapy for trichiasis greatly. It is also an advantage to be able to use a nitrous oxide probe, as this type of machine is now widely available and is more convenient than other methods of cooling. It should be noted that the retinal cryoprobe does not have a sufficiently high cooling rate to provide fast enough freezing within the lid tissue. Other workers ${ }^{67}$ using this method, with or without temperature monitoring, have shown a lower success rate.

We have used cryotherapy on as many as three occasions to the same area of the lid without an increase of complications. Wood and Anderson, ${ }^{8}$ who reported a high incidence of complications, also treated lids on up to four occasions but did not relate the complication rate to the number of applications. The low number of complications in our study may be due to the method used or possibly to the initial absence of associated conjunctival disease in our patients.

This method of treatment was very acceptable to our group, and only one patient refused a second application. Treatment was given as an outpatient procedure on the day that the patient was seen in clinic. As well as being convenient to patients, expensive hospital admissions and surgery can be avoided.

\section{References}

1 Sullivan JH, Beard C, Bullock JD. Cryosurgery for treatment of trichiasis. Am J Ophthalmol 1976; 82: 117-21.

2 Johnson RLC, Collin JRO. Treatment of trichiasis with a lid cryoprobe. Br J Ophthalmol 1985; 69: 267-70.

3 Hecht SD. Cryosurgery of trichiasis with use of the retinal cryoprobe. Am J Ophthalmol 1977; 90: 1501-3.

4 Fraunfelder FT, Farris HE, Wallace TR, et al. The role of cryosurgery in external ocular and periocular disease. Ophthalmology (Rochester) 1977; 83: 713-24.

5 Collin JRO. Manual of systematic eyelid surgery. London: Churchill-Livingstone, 1983.

6 Collin JRO, Cosler DJ, Sullivan JH. Cryosurgery for trichiasis. Trans Ophthalmol Soc UK 1978; 98: 81-3.

7 Majekodunmi S. Cryosurgery in treatment of trichiasis. $\mathrm{Br} \mathrm{J}$ Ophthalmol 1982; 66: 337-9.

8 Wood JR, Anderson RL. Complications of cryosurgery. Arch Ophthalmol 1981; 99: 460-3.

Accepted for publication 16 December 1985. 